\title{
Idiopathic atrial flutter AFL in neonates (Case Report)
}

\author{
Saimir Kuci ${ }^{1}$, Alfred Ibrahimi ${ }^{1}$, Stavri Llazo ${ }^{1}$, Ervin Bejko ${ }^{1}$, Jonela Burimi ${ }^{1}$, Esmerilda Bulku ${ }^{1}$ \\ 1. "Mother Teresa" Hospital, Cardioanesthesy Services, Rr. Dibres 372 Tirana, Albania \\ E-mail of the corresponding author: saimirkuci@gmail.com
}

\begin{abstract}
Atrial flutter AFL is a rare arrhythmia in the neonate and early infants. Here we report the case of a term female infant (A.K), with a birth weight of $3000 \mathrm{~g}$, who was delivered by Cesarian section of a 26 -year-old primiparous mother (I.K) in "Queen Geraldine" Maternity in Tirana. The APGAR scores were 9 and 10 at $1^{\text {st }}$ and $5^{\text {th }}$ minute, respectively. In prenatal history, no fetal arrhythmia was present. After waters breaks, a fetal arrhythmia was detected. Then the infant was delivered by an urgent $\mathrm{C}$ section. The infant was treated by medical treatment, which resulted unsuccessful. In the $16^{\text {th }}$ day of life, the DC shock was applied and a sinus rhythm was detected immediately.
\end{abstract}

Neonate: A.K Mother: I.K

Keywords: infant, atrial flutter, antiarrhythmic, DC shock

DOI: $10.7176 /$ ALST/80-05

Publication date: July $30^{\text {th }} 2020$

\section{Introduction}

1.1 What is atrial flutter?

Atrial flutter AFL refers to an arrhythmia, or an abnormal heart rhythm that involves an electrical circuit formed in the atrium, the upper two chambers of the heart. Normal electrical conduction in the heart starts with the generation of electricity in the sinus node in the upper portion of the right atrium. Electricity moves from the sinus node through the atrium. From there, it is transmitted to the AV node to the ventricles. As electricity passes to the ventricles, the heart muscle contracts.Atrial flutter occurs when electricity forms an abnormal circuit somewhere in the atrium. As electricity moves around the circuit, it generates impulses at a very fast rate. The sinus node is completely shut down in this situation. Usually the heart rate in the atrium is very fast, often in the range of 350-500 beats per minute. The AV node is usually incapable of transferring impulses at such a fast rate, so only every other or every third impulse is conducted to the ventricles. This results in a heart rate in the ventricles that is much slower than the atrium, but still usually faster than normal. For example, if the atrium are beating at 360 beats per minute, and only every third beat gets through to the ventricles, the ventricles will beat at 120 beats per minute.

\subsection{What causes atrial flutter in children?}

Atrial flutter in children is seen most commonly in newborn babies. In this setting, it is usually caused by the relative immaturity of the heart's electrical system. It is usually noticed when somebody listens and hears a faster than normal heart rate in an otherwise healthy appearing baby. Although medication may be effective, usually the quickest and most efficient means of treating atrial flutter in a baby involves an electrical cardioversion. In this setting, an electrical shock is delivered to the baby's chest. This momentarily stuns was the heart and resets the electricity completely. The atrial flutter circuit is terminated and the sinus node is able to effectively take over again. Atrial flutter in babies is usually a one-time problem and typically does not recur. Most babies who have atrial flutter are observed for at least 48 hours. If there is no recurrence of the problem during that time frame, it is usually safe to discharge the baby without any medication. Recurrence is very unusual.

Atrial flutter in older children is very uncommon. When it does occur, it is almost always in the setting of surgically repaired heart disease. Scar tissue caused by surgery can create a circuit in which atrial flutter may occur. In this setting, atrial flutter is again treated most effectively with electrical cardioversion. However, as opposed to babies, the likelihood of recurrence is relatively high. Most older children and teenagers who develop atrial flutter require long-term medication.

\subsection{What are the signs and symptoms of atrial flutter?}

The danger of atrial flutter, or any tachycardia for that matter, involves the degree of stress placed on the heart muscle. When the heart rate is very fast, or even when the heart rate is not so fast but continues for a prolonged period of time, the heart muscle tires out. This may lead to ineffective pumping and the development of congestive heart failure. Symptoms of congestive heart failure in babies include rapid breathing, sweating, poor feeding, and poor weight gain. In older children, atrial flutter usually causes symptoms of palpitations. Rarely associated symptoms of chest pain, dizziness, or syncope may occur as well. 


\subsection{How is atrial flutter treated?}

The most effective treatment of atrial flutter is electrical cardioversion. In babies this usually is a one-time treatment that solves the problem permanently. In older children, medication is often necessary to prevent recurrence.

In summary, atrial flutter in children is relatively uncommon. Fortunately most cases are treatable with either electrical cardioversion or medication, or a combination of the two.

\subsection{Epidemiology}

AFL is rare with an approximate incidence of around 1/50,000 live births in Europe.

\subsection{Clinical description}

AFL has a neonatal onset and the majority $(72 \%)$ of patients present with symptoms within the first $48 \mathrm{~h}$ of life. Onset during the fetal period may be associated with hydrops fetalis and death. AFL manifests with tachycardia and congestive heart failure. AFL is sustained although conversion from atrial flutter to sinus rhythm may occur spontaneously $(<24$ hours). Some patients are asymptomatic and tachycardia may be noted on routine examination and monitoring.

1.7 Etiology

The etiology of AFL is unknown but immaturity of the myocardium and the high pressure in the right atrium during the perinatal period may be factors that favor the appearance of atrial re-entry.

\subsection{Diagnostic methods}

The diagnosis of AFL relies on surface electrocardiogram (ECG) which usually shows an atrial rate of around 440 beats/min, most often with 2:1 atrioventricular conduction (sometimes with variable AV conduction and an irregular ventricular rate of 125-280 beats/min), and saw tooth $\mathrm{P}$ waves in leads II, III, and aVF. The echocardiogram may show impaired ventricular function after prolonged tachycardia.

Differential diagnosis

Differential diagnosis includes multifocal atrial tachycardia (see this term) and other forms of supraventricular tachycardia.

\subsection{Antenatal diagnosis}

AFL may be diagnosed prenatally by fetal echocardiogram which will show an atrial rate of over 400 per minute and most often 2:1 AV conduction to give a ventricular rate of over 200 per minute.

\subsection{Management and treatment}

The first aim of treatment is the restoration of sinus rhythm. This is most easily achieved by transoesophageal overdrive pacing or synchronised low energy DC cardioversion using around $0.5-1 \mathrm{~J} / \mathrm{kg}$. The response to drug treatment is variable and unpredictable. Recurrence of atrial flutter in neonates is rare and prophylactic medication is usually not required. Sotalol is said to be an effective agent in the treatment of AFL in the fetus as it has excellent transplacental passage.

\subsection{Prognosis}

In the absence of additional arrhythmias, infants with AFL have an excellent prognosis once in sinus rhythm, with a low risk of recurrence, and chronic anti arrhythmic therapy is unlikely to be necessary.

\section{Case Presentation}

A term (39 weeks; 2 days), female infant (A.K) was born by an urgent C section from the first pregnancy of a 26-year-old-mother (I.K) with no concomitant diseases, in "Queen Geraldine" Maternity. At the physical examination tachycardia (HR $>200 / \mathrm{bpm}$ ) was examined. Her birth weight was $3000 \mathrm{~g}$. As ECG detected Atrial Flutter, intravenous amiodarone was started. In the fifth day, propanolol was added to therapy. As AFL remained resistant to these therapies, a combination of intravenous amiodarone and oral flecainide therapy was initiated. After five days of amiodarone and flecainide, at the $16^{\text {th }}$ day of follow up, cardioversion was performed in "Mother Teresa" Hospital, Cardioanesthesy \& Pediatric Services. Once the DC shock was performed, normal sinus rhythm was detected immediately. In the following days, the therapy continued with flecainide p.os.

The patient was discharged at the $18^{\text {th }}$ day, sinus rhythm present.

Term (39 weeks; 2 days), AGA (Appropriate for Gestational Age)

Apgar score: $1^{\text {st }}$ minute: $9 ; 5^{\text {th }}$ minute: 10

Birth weight: $3000 \mathrm{~g}$; Body length: $50 \mathrm{~cm}$

Other parameters: blood pressure 52/25 mmHg, SpO2 98 \%, RR 55-58 /rpm 


\subsection{Echocardiogram:}

$2^{\text {nd }}$ day of life Patent Foramen Ovale and Ductus Arteriosus (begins to close)

$5^{\text {th }}$ day of life Foramen Ovale present

$9^{\text {th }}$ day of life Foramen Ovale present

The heart valves open and close properly. There are not leaking blood. Heart walls and chambers are normal in size and the heart wall muscles move properly. No DIA or DIV present. Other parameters are in range.

\subsection{Therapy:}

$1^{\text {st }}$ day: Amiodarone $5 \mathrm{mg} / \mathrm{kg}$ during $30 \mathrm{~min}$, following hours $10 \mathrm{mg} / \mathrm{kg}$

$5^{\text {th }}$ day: Amiodarone iv + Propanolol $3 \times 1 \mathrm{ml}$ p.os (added to therapy)

$9^{\text {th }}$ day: Ampiciline + Gentamicine + Ceftazidime (added to therapy due to phlebitis)

$13^{\text {th }}$ day: Amiodarone iv + Flecainide $2 \times 2 \mathrm{ml}$ p.os (added to therapy). Propanolol is stopped.

$16^{\text {th }}$ day: DC shock, following days Flecainide $3 \mathrm{mg} / \mathrm{kg} /$ day p.os ,Fig
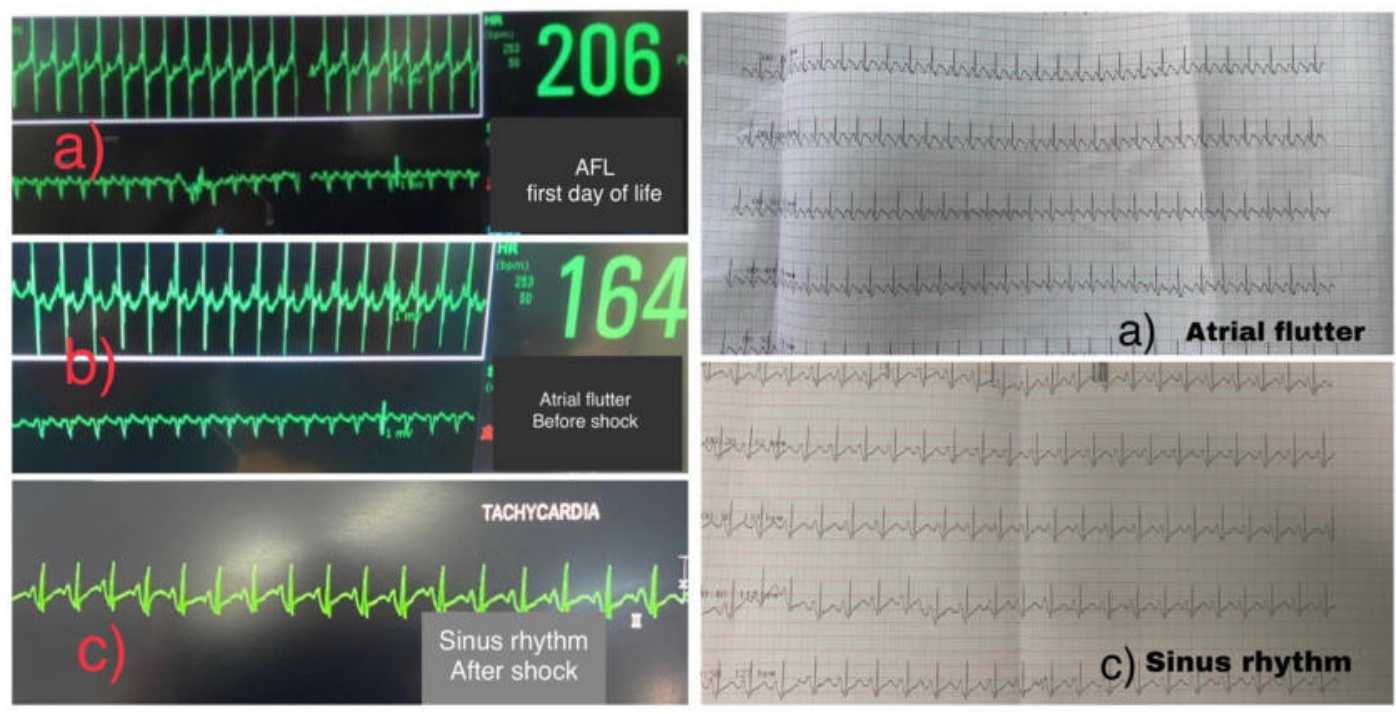

a)Atrial flutter, first day of life

b)Atrial flutter, before DC shock

c)Sinus rhythm, after shock

\section{Discussion}

Atrial flutter is the most commonly reported tachyarrhythmia in the fetal period, being rare in its isolated form in the neonatal period 1 Its etiology is uncertain, but there is an association with structural heart diseases, which should be promptly ruled out by echocardiography. The presence of structural alterations is correlated with worse prognosis. ${ }^{2}$

The diagnosis is often simple, with the electrocardiogram showing the typical "sawtooth" or "picket fence" pattern, better observed in leads II, III and aVF. The clinical presentation of atrial flutter depends on the ventricular response to atrial tachyarrhythmia. In newborns, the atrial rate is 400 beats per minute (bpm), with an atrioventricular conduction ratio of $2: 1$, resulting in a ventricular rate of approximately $200 \mathrm{bpm} .{ }^{-}$The patient described had a ventricular rate of around 190-230bpm and atrial rate of 380-460bpm.

Complications related to the umbilical venous catheterization are reported regarding its inadequate positioning..$\underline{-}$ ${ }^{4}$ There are methods to determine the correct insertion length of umbilical venous catheters. $\frac{5-6}{}$ One approach uses

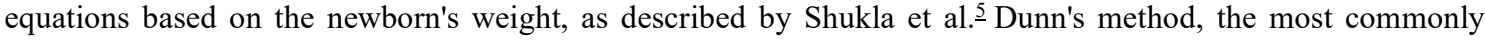
used one, is based on the measurement of the shoulder-navel distance. This method is hindered by numerous important limitations, including interpersonal variations. ${ }^{-}$It is not known whether the estimated insertion length based on these methods is accurate. ${ }^{?}$ Once the patient is catheterized, the location is routinely verified by a chest $\mathrm{X}$-ray. In chest X-rays in the anteroposterior view, the ideal position for the catheter tip is located between the 
T8 and T9 thoracic vertebrae. In this position, $90 \%$ of the catheters will have their distal end placed between the right atrium and the inferior vena cava.

It is noteworthy that $56 \%$ of umbilical venous catheters, when radiography was used to verify the location of the catheter tip, had to be repositioned because they were placed in an unsatisfactory site. $\frac{8-9}{}$ When comparing the methods to identify the correct position of catheters, ultrasound is a more accurate complementary method of examination than chest X-ray to determine the route and the position of venous umbilical catheters. $\frac{8-9}{}$ The first difficulty is to cross the ductus venosus and then reach its center position. This difficulty justifies the complications related to poor positioning. ${ }^{8}$ In the patient described in this report, the Dunn method was used as a reference for catheter positioning, with its position being assessed by chest X-ray, according to the service routine.

The association between diabetic mothers and newborns with atrial arrhythmias has been reported in the literature. Some studies have shown that fetuses and neonates with atrial tachyarrhythmias are most commonly large for gestational age or the offspring of diabetic mothers, as the patient shown here. They have cardiac function alterations, regardless of the presence of ventricular hypertrophy. There is the hypothesis that diastolic dysfunction, with subsequent atrial dilation, may predispose infants of diabetic mothers to atrial arrhythmias. ${ }^{-}$

The management of newborns with cardiac arrhythmias secondary to umbilical venous catheterization has not been systematically studied. As arrhythmias often occur due to poor positioning of the catheter, the first step should be to pull it back or even remove the catheter. However, in our case, catheter pullback was not effective. Treatment to bring the heart back to sinus rhythm can be pharmacological (antiarrhythmics such as adenosine, digoxin, amiodarone, etc.) or electric (cardioversion or transesophageal atrial stimulation). Adenosine administration may be effective, but it does not always treat tachycardia of atrial origin, such as atrial flutter, as seen in the patient described here. $\frac{10-11}{1}$ However, adenosine administration can help identify atrial flutter, as it produces a transient atrioventricular block, demonstrating the arrhythmia-characteristic wave in the ECG. $\frac{10-}{}$ 12 New drugs, most of which have already been used in adults and children, are being studied, such as ibutilide and propafenone, and there have been reports of their use in newborns. $\frac{13-14}{}$ When there is no response to pharmacological treatment, therapy may require synchronized cardioversion or transesophageal atrial pacing, with high probability of sinus rhythm conversion. $\frac{1-13-14}{1 n}$ cases with hemodynamic instability, electrical cardioversion should be preferably used, as in the case reported here.

Leroy et al. $\underline{5}$ described a similar case, in which a full-term newborn developed atrial flutter after an umbilical venous catheter was placed in the left atrium. Treatment consisted in repositioning the catheter and transesophageal atrial pacing, with good evolution. Sinha et al. ${ }^{7}$ also reported a patient with hemodynamic instability due to atrial flutter after catheterization, resolved after synchronized cardioversion.

Fetal and neonatal atrial flutter is associated with significant morbidity. However, mortality seems to be more

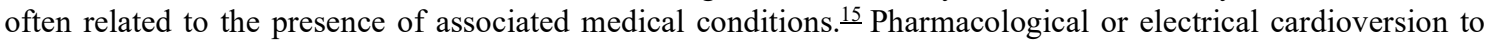
normal sinus rhythm may be effective and, once such reversal is achieved, the patient does not seem to have a risk of recurrence of atrial flutter, except when there is an accessory pathway; in this case, supraventricular tachycardia episodes may occur. In patients without an accessory pathway, it is not usually necessary to maintain the long-term medication. $\frac{15-16}{}$

Considering the small amount of data available in the national and international literature, mainly from the isolated case reports, there is scarce information about the precise association between neonatal arrhythmias and the umbilical catheterization, including the actual incidence of atrial flutter and the indication of a "universal" therapeutic approach for it. The association between cardiac arrhythmias and maternal diabetes is a research field yet to be developed, which can contribute to the prevention of this disease, supported by a better understanding of the physiopathological mechanisms of these entities.

\section{Conclusion}

Atrial flutter is the most commonly reported tachyarrhythmia in the fetal period, being rare in its isolated form in the neonatal period. Its etiology is uncertain, but there is an association with structural heart diseases, which should be promptly ruled out by echocardiography. The presence of structural alterations is correlated with worse prognosis. Treatment to bring the heart back to sinus rhythm can be pharmacological antiarrhythmics such as adenosine, digoxin, amiodaron .Some time is indication transoesophageal overdrive pacing or synchronised low energy DC cardioversion using around $0.5-1 \mathrm{~J} / \mathrm{kg}$. 


\section{References}

1. Mendelsohn A, Dick M 2nd, Serwer GA. Natural history of isolated atrial flutter in infancy. J Pediatr. 1991;119:386-91. [ Links ]

2. Lisowski LA, Verheijen PM, Benatar AA, Soyeur DJ, Stoutenbeek P, Brenner JI, et al. Atrial flutter in the perinatal age group: diagnosis, management and outcome. J Am Coll Cardiol. 2000;35:771-7. [ Links ]

3. Hermansen MC, Hermansen MG. Intravascular catheter complications in the neonatal intensive care unit. Clin Perinatol. 2005;32:141-56. [ Links ]

4. Ramasethu J. Complications of vascular catheters in the Neonatal Intensive Care unit. Clin Perinatol. 2008;35:199-222. [ Links ]

5. Leroy V, Belin V, Farnoux C, Magnier S, Auburtin B, Gondon E, et al. Une observation de flutter auriculaire après pose de cathéter veineux ombilical. Arch Pediatr. 2002;9:147-50. [ Links ]

6. Dunn PM. Localization of the umbilical catheter by post-mortem measurement. Arch Dis Child. 1966;41:69-75. [ Links ]

7. Verheij GH, Te Pas AB, Witlox RS, Smitis-Wintejens VE, Walther FJ, Lopriore E. Poor accuracy of methods currently used to determine umbilical catheter insertion length. Int J Pediatr. 2010;33:78-83. [ Links ]

8. Greenberg M, Movahed H, Peterson B, Bejar R. Placement of umbilical venous catheters with use of bedside real-time ultrasonography. J Pediatr. 1995;126:633-5.[ Links

9. Michel F, Brevaut-Malaty V, Pasquali R, Thomachot L, Vialet R, Hassid S, et al. Comparison of ultrasound and X-ray in determining the position of umbilical venous catheters. Resuscitation. 2012;83:705-9. [ Links ]

10. Clarke B, Till J, Rowland E, Ward DE, Barnes PJ, Shinebourne EA. Rapid and safe termination of supraventricular tachycardia in children by adenosine. Lancet. 1987;1:299-301. [ Links ]

11. Till J, Shinebourne EA, Rigby ML, Clarke B, Ward DE, Rowland E. Efficacy and safety of adenosine in the treatment of supraventricular tachycardia in infants and children. Br Heart J. 1989;62:204-11. [ Links ]

12. Dixon J, Foster K, Wyllie J, Wren C. Guidelines and adenosine dosing in supraventricular tachycardia. Arch Dis Child. 2005;90:1190-1. [ Links ]

13. Konak M, Alp H, Tarakçı N, Baysal T, Örs R. Successful treatment of atrial flutter with propafenone and synchronized cardioversion in a newborn. Indian J Pediatr. 2014;81:413-4. [ Links ]

14. Prasad D, Snyder C, Ashwath R. Ibutilide therapy in atrial flutter conversion in neonates. Heart Rhythm. 2013;10:1231-3. [ Links ]

15. Barclay RP, Barr DG. Direct current cardioversion in a case of congenital atrial flutter. Arch Dis Child. 1972;47:833-4. [ Links ]

16. Casey FA, McCrindle BW, Hamilton RM, Gow RM. Neonatal atrial flutter: significant early morbidity and excellent long-term prognosis. Am Heart J. 1997;133:302-6. [ Links ] 